\title{
Development of Piezoelectric Bending Actuators with Embedded Piezoelectric Sensors for Micromechanical Flapping Mechanisms*
}

\author{
Domenico Campolo, Ranjana Sahai, Ronald S. Fearing \\ \{minmo, rsahai, ronf $\} @$ eecs.berkeley.edu \\ Department of EECS, University of California, Berkeley, CA 94720. USA
}

\begin{abstract}
This paper presents the fabrication and the testing of piezoelectric unimorph actuators with embedded piezoelectric sensors which are meant to be used for the actuation of the Micromechanical Flying Insect (MFI). First the fabrication process of a piezoelectric bending actuator comprising a standard unimorph and a rigid extension is described together with the advantages of adding such an extension. Then the convenience of obtaining an embedded piezoelectric sensor by a simple and inexpensive variation of the fabrication process is pointed out. A model for the sensor embedded into a unimorph actuator with rigid extension is derived together with its flat response band limits. Calibration steps are also outlined which allow, despite residual parasitic actuator-sensor coupling, the use of the actuator with the embedded sensor for measuring position and inertial forces when external mechanical structures are driven. An experiment is carried out which validates the model for the actuator/sensor device under desired operating conditions. Preliminary application of the fabricated device to the MFI is also presented where the mechanical power fed into the wing is estimated.
\end{abstract}

\section{Introduction}

Piezoelectric actuators are widely used in smart structure applications due to their high bandwidth, high output force, compact size, and high power density properties. For such reasons they are very appealing for mobile microrobotic applications such as the Micromechanical Flying Insect (MFI) [1] where, because of strict size/weight constraints, smart structures capable of both actuating and sensing are preferred. Since the technology needed to fabricate PZT based bending actuators was already available [4], the

\footnotetext{
*This work was funded by ONR MURI N00014-98-1-0671, DARPA.
}

possibility of integrating sensorial capabilities into the actuators themselves was investigated. Many works exist where piezoelectric thin patches are bonded to structures in order to sense the deformation of a specific area [2] but, in most of the cases, the process for fabricating unimorph actuators needs to be heavily modified. The idea of extending the capabilities of a standard unimorph actuator with a lateral sensor came from [6] although no hint was given for the fabrication involved.

The possibility of having the sensing section and the actuating section coexisting on the same piezoelectric layer, differentiated by simply patterning the electrode instead of aligning and bonding two different piezoelectric layers next to one another, was investigated. The sensor obtained in this way was affected by a strong electromechanical coupling between the actuating area and the sensing area, mainly a parasitic capacitance between the two electrodes. In order to shield such a capacitance, a third grounded electrode was then introduced in between the two sections.

\section{$2 \quad$ Fabrication}

Since the publication of [4], several improvements have been made to the fabrication of PZT based unimorph actuators. The only parameter unchanged is the ratio of thicknesses of the two layers constituting the unimorph since its choice was based upon the output energy optimization criteria [4].

The basic unimorph is obtained by bonding together stainless steel and PZT (PZT-5H, T105-H4E-602 ceramic single sheet, Piezo Systems, Inc.) respectively with thicknesses $t_{s}=76.2 \mu \mathrm{m}$ and $t_{p}=127 \mu \mathrm{m}$. Fabrication details can be found in [4].

A different commercial piezoelectric material (PZNPT from TRS Ceramics, Inc.) will be eventually used for the actuation of the MFI because of its superior properties [4]. PZN-PT is a single crystal piezoelec- 


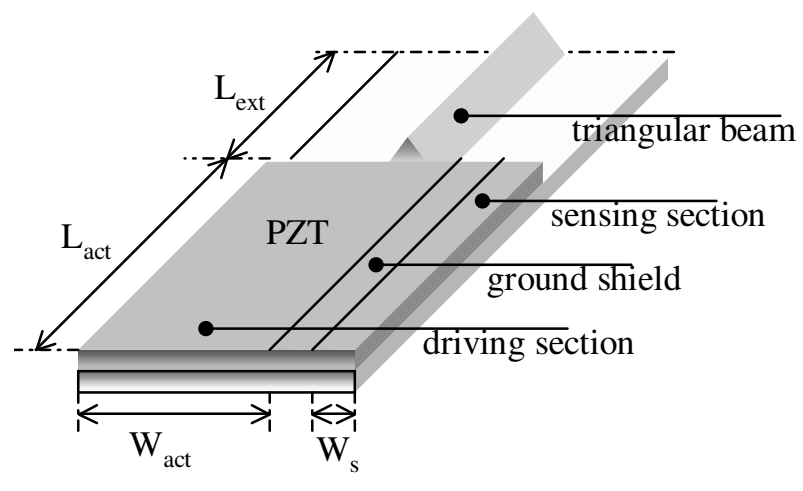

Figure 1: Piezoelectric actuator diagram comprising basic unimorph actuator and rigid extension.

tric material which is available in fixed dimensions. Difficulties arising in reshaping its size, without damaging its single crystal properties, impose the use of its original dimensions. On the contrary, PZT is a ceramic material, relatively inexpensive, which can be simply reshaped by laser cutting without perceptible loss of its properties. Although much larger, for testing purposes, an equivalent unimorph can be made out of PZT which provides, from the mechanical output, the same stiffness and blocking force theoretically provided by a PZN-PT based unimorph. Planar dimensions for the PZT based unimorph have been determined to be (width) $W_{a c t}=6 \mathrm{~mm}$ and (length) $L_{a c t}=12 \mathrm{~mm}$.

\section{$2.1 \quad$ Rigid Extension}

MFI [4] is a biomimetic project and a major design constraint is the wing beat resonance, determined to be at about $150 \mathrm{~Hz}$. The stiffness of the actuator is therefore designed [4] to resonate, together with wing inertia reflected through an amplifying mechanism (4-bar mechanism), at this frequency. With the given fixed dimensions for the PZN-PT based unimorph, which translate into $W_{a c t}, L_{a c t}, t_{s}$ and $t_{p}$ for the PZT based equivalent one, the resulting stiffness would be too large. A rigid extension can be designed so that, by acting as a lever, it would provide larger free displacement at its tip together with lower blocking force, thus, leading to lower stiffness. In order to obtain the required stiffness, a rigid extension of length $L_{e x t}=8 \mathrm{~mm}$ is needed. Rigidity of such an extension is a necessity. A flexible one would bend, storing part of the actuation energy instead of transmitting it to the wing. A first idea would be extending the very stainless steel layer which constitutes the uni- morph but it can be easily seen that it would be even more compliant than the unimorph itself. A stiffer structure can be bonded on top of the extended stainless steel such as a hollow triangular beam as shown in Fig. 1. Such a folded structure is much stiffer than a planar structure. It can in fact be made out of thinner, therefore lighter, stainless steel $(12.5 \mu \mathrm{m})$ and still be considered rigid. The lighter the extension the more ideal it can be considered. As shown later, the inertia of the reflected wing is about $190 \mathrm{mg}$ while the inertia of the extension is less than $10 \mathrm{mg}$. The dynamics, i.e. the inertial terms, of the extension can then be neglected.

Advantages of adding a light and hollow rigid extension instead of having a longer unimorph with similar output mechanical behavior, i.e. stiffness and blocking force at the tip, can be summarized as:

- the final actuator will be lighter since a hollow beam replaces a comparably long section of the unimorph itself.

- higher first mode resonant frequency since also the equivalent mass of the actuator, which together with its stiffness defines the first resonance, is lighter.

- behaving as a lever, the rigid extension represents a first stage where the actuator tip displacement is pre-amplified. A pre-amplifying stage helps reducing nonlinearities deriving from a single high ratio amplifying stage (often needed in piezoelectric actuators based applications).

- higher energy density can be achieved since the extension converts a force load at the tip into a combination of the same force plus a torque (see Fig. 5) at the unimorph tip. Such a combination produces more bending throughout the unimorph than only the force would.

\section{$2.2 \quad$ Side Sensor}

As qualitatively described in [6], considering an actuator of width $W_{\text {act }}$ and length $L_{a c t}$ constituted by a piezoelectric layer bonded onto an elastic layer, another narrow piezoelectric strip of width $W_{s} \ll W_{a c t}$ and same length as the actuator can be bonded onto the elastic layer, side by side with the piezoelectric layer constituting the unimorph actuator itself. This way it will be subject to the same deflection as the actuator and via the piezoelectric effect a measure of the mean curvature of the actuator can be derived, as shown later. 


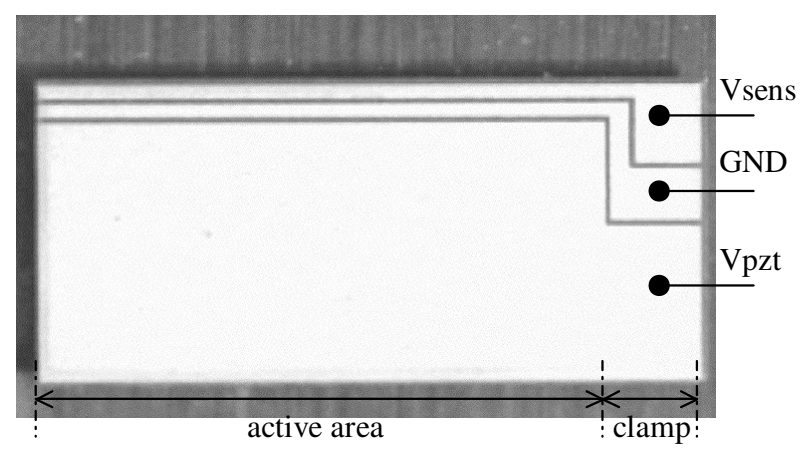

Figure 2: A photo the piezoelectric layer after laser cutting and top electrode patterning.

The sensor should be much narrower than the actuator in order not to affect the deflection. Ideally there should be no coupling between the piezoelectric layer of the unimorph and the sensing strip.

PZT ceramics can be easily shaped by laser cutting. A $532 \mathrm{~nm}$ (green) laser beam can be generated by the QuikLaze micro-machining system (New Wave Research Inc.) which, at its maximum output power, is capable of cutting through PZT quickly enough not to overheat the sample. This allows precisely cutting out the piezoelectric pieces that will be lately bonded onto the stainless steel layer. By reducing the power of the laser beam, it is also possible to pattern the very thin layer of nickel that constitutes the electroding layer of the piezoelectric sheets. Since laser cutting the PZT pieces is a necessary step in the fabrication of the actuator, adding electrode patterning to the current process is a very inexpensive operation since no alignment has to be done and it takes relatively little time compared with the laser cutting itself.

Fig. 2 shows a photo of the piezoelectric layer whose top side electrode has been patterned with low power laser beam and then cut through with high power laser beam. Its whole length is divided into an active area which will be free to bend together with the elastic layer and the remaining area which, together with the elastic layer, will be clamped and kept from bending. The clamping area is where the wires will contact the electrodes, $V_{p z t}$ for the actuator and $V_{\text {sens }}$ for the sensor. The presence of a third electrode $(G N D)$ is explained below.

Differently from previous work [2], in order to keep the fabrication process simple and inexpensive, the actuator and the sensor coexist on the same piece of piezoelectric material. They only differ from one another because of the discontinuity of the electrodes,

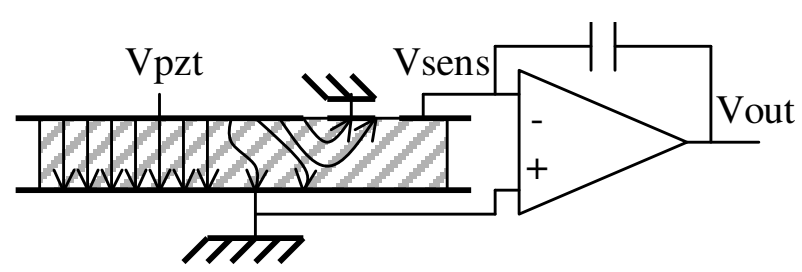

Figure 3: Front view ( $x y$ plane) of the piezoelectric layer and its electrical connection.

leading thus to a strong electromechanical coupling. As shown later, the sensor is meant to reveal a structural bending by producing polarization charge within its volume, proportional to the mean curvature of the unimorph actuator. An electromechanical coupling with the actuator section leads to extra induced charge proportional to the driving voltage and always in phase with it (at least at the frequencies of interest) while the structural bending would not always be in phase with the driving voltage because of the structural modes. The parasitic coupling will therefore result into an offset signal, also called feed-through, always in phase with the driving voltage and that can be taken into account during the calibration. Before calibrating, however, an effective way of shielding the electrical coupling is possible by introducing an electrically grounded strip between actuator and sensing section (referred to as GND in Fig. 2) which can act as a shield for the electric field line coming from the actuator section.

Fig. 3 shows electrical connections of the piezoelectric device with driving voltage $V_{p z t}$ and the charge amplifier while the bottom electroded and the top shielding strip are electrically grounded. Because of the operational amplifier, the sensing electroded is also at virtual ground. The electric field in the piezoelectric layer of a standard unimorph actuator with a single top electrode can be considered vertical ( $z$ direction) but when a patterned electrode is considered, as in the case of interest, the field fringing can become significant. Fig. 3 schematically sketches how the most of the electric field lines are captured by the grounded strip, thus shielding the sensing area.

\section{Model of a Unimorph Actua- tor Plus Rigid Extension}

A working model for the unimorph actuator can be derived from [7]. Following [7], a coordinate system is 


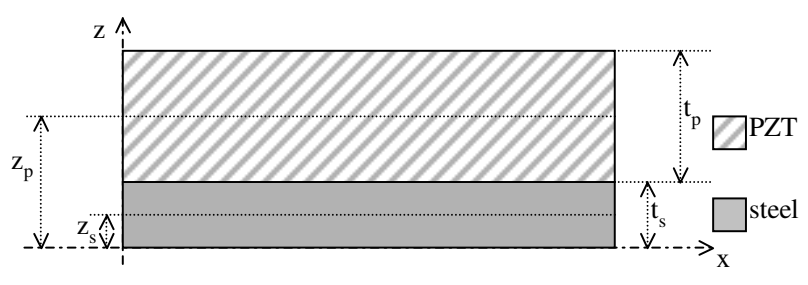

Figure 4: Coordinate system: the unimorph actuator is a long, narrow and uniform beam along the $x$ axis. Non uniformity, i.e. different materials, is only assumed along the $z$ direction.

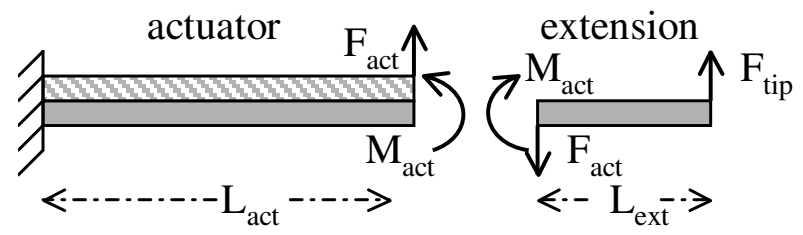

Figure 5: Free body diagram for the unimorph actuator (left) plus rigid extension (right).

adopted as in Fig. 4 such that $z=0$ coincides with the base of the beam. Let $z_{p}$ and $z_{s}$ be respectively the coordinate of the center of piezoelectric and stainless steel layer, $t_{p}$ and $t_{s}$ their thicknesses, $Y_{p}$ and $Y_{s}$ be their Young's modulus. The neutral axis can be defined as (equation (11) in [7]):

$$
z_{n}=\frac{z_{s} Y_{s} t_{s}+z_{p} Y_{p} t_{p}}{Y_{s} t_{s}+Y_{p} t_{p}}
$$

while the moment of inertia is:

$$
\begin{aligned}
E I= & W_{a c t} Y_{s}\left(\left(z_{s}-z_{n}\right)^{2} t_{s}+\frac{t_{s}^{3}}{12}\right) \\
& +W_{a c t} Y_{p}\left(\left(z_{p}-z_{n}\right)^{2} t_{p}+\frac{t_{p}^{3}}{12}\right)
\end{aligned}
$$

When bending occurs, $z_{n}$ will actually depend upon $x$ (the coordinate system is in fact defined upon the rest configuration of the unimorph) and also $z_{s}$ and $z_{p}$ but not $\left(z_{s}-z_{n}\right)$ nor $\left(z_{p}-z_{n}\right)$ which will be constant. Referring to the left side of Fig. 5, equation (26) in [7] can be reduced to the case where $\delta_{a c t}$ and $\alpha_{a c t}$, respectively deflection and slope at the tip of the actuator, are to be determined as linear functions of $F_{a c t}$ and $M_{a c t}$. Moreover, the effect of an applied voltage $V_{p z t}$ can be seen as due to an equivalent mechanical moment $M_{p z t}=M_{V} V_{p z t}$ applied at the tip of the actuator, just like $M_{a c t}$, where $M_{V}$ is the equivalent mechanical torque per unit voltage at the electrodes [7]. The following equation holds:

$$
\left[\begin{array}{ll}
\delta_{a c t} & \alpha_{a c t}
\end{array}\right]^{T}=\mathbf{S}_{\mathrm{act}}\left(\left[\begin{array}{ll}
F_{a c t} & M_{a c t}
\end{array}\right]^{T}+\left[\begin{array}{ll}
0 & M_{p z t}
\end{array}\right]^{T}\right)
$$

where:

$$
\mathbf{S}_{\text {act }}=\frac{L_{a c t}}{E I}\left[\begin{array}{cc}
\frac{L_{a c t}^{2}}{3} & \frac{L_{a c t}}{2} \\
\frac{L_{a c t}}{2} & 1
\end{array}\right]
$$

is the compliance matrix of the unimorph actuator.

Equation (3) simply assumes that the actuator operates at quasi-static conditions. Such an assumption is valid for frequencies much below the first resonant mode which, in the case under consideration, occurs after $500 \mathrm{~Hz}$ while the working frequencies are at about $150 \mathrm{~Hz}$.

As for the rigid extension in the right side of Fig. 5, the dynamical equations of a rigid body simply transform into a lever equation:

$$
\left[\begin{array}{ll}
F_{\text {act }} & M_{a c t}
\end{array}\right]^{T}=\left[\begin{array}{ll}
1 & L_{e x t}
\end{array}\right]^{T} F_{\text {tip }}
$$

if inertial terms are negligible. Equation (5) combined with (3) leads to:

$$
\left[\begin{array}{ll}
\delta_{a c t} & \alpha_{a c t}
\end{array}\right]^{T}=\mathbf{S}_{\text {act }}\left(\left[\begin{array}{ll}
1 & L_{\text {ext }}
\end{array}\right]^{T} F_{t i p}+\left[\begin{array}{ll}
0 & M_{p z t}
\end{array}\right]^{T}\right)
$$

As previously mentioned $M_{p z t}=M_{V} V_{p z t}$, i.e. after calibration, $M_{p z t}$ can be directly derived by measuring the input voltage. The sensor, as shown later, will provide a measurement of $\alpha_{\text {act }}$. Therefore, after algebraic manipulation, (6) can be rewritten as:

$$
\left[\begin{array}{lll}
\delta_{a c t} & F_{t i p}
\end{array}\right]^{T}=\mathbf{G}_{\text {act }}\left[\alpha_{a c t} V_{p z t}\right]^{T}
$$

moreover, $\delta_{t i p}$, i.e. the displacement at the tip of the extension, is easily evaluated as:

$$
\delta_{t i p}=\left[\begin{array}{ll}
1 & L_{e x t}
\end{array}\right] \cdot\left[\begin{array}{ll}
\delta_{a c t} & \alpha_{a c t}
\end{array}\right]^{T}=\delta_{a c t}+\alpha_{a c t} L_{e x t}
$$

\section{Model of Piezoelectric Sensor}

The model for the charge across the grounded electrodes of a narrow strip of piezoelectric material bonded to a cantilevered beam subjected to a given nonuniform curvature is derived here similarly to [7]. Considering only the piezoelectric material, let $S$ and $T$ be respectively the $x$-axis components of the mechanical strain and stress and $E$ and $D$ be respectively the $z$-axis components of the electric field and the dielectric displacement. The other components are not considered, as in [7]. The constitutive piezoelectric equations are:

$$
\left\{\begin{aligned}
S & =\left(Y_{p}^{E}\right)^{-1} T+d_{31} E \\
D & =d_{31} T+\epsilon^{T} E
\end{aligned}\right.
$$

where $Y_{p}^{E}$ is the Young's modulus at constant electric field, $d_{31}$ is piezoelectric coefficient and $\epsilon^{T}$ is the dielectric permittivity at constant stress. The first equation 
of (9) can be substituted into the second leading to:

$$
D=d_{31} Y_{p}^{E} S+\left(\epsilon^{T}-Y_{p}^{E} d_{31}^{2}\right) E
$$

As in [5] and [7], the strain is directly related to the neutral axis $z_{n}(x)$ by:

$$
S=-\left(z-z_{n}\right) \frac{\partial^{2} z_{n}(x)}{\partial x^{2}}
$$

Integrating (10) along $z$ from one electrode located at $z_{1}=z_{p}-t_{p} / 2$ to the other located at $z_{2}=z_{p}+t_{p} / 2$ and considering that $\int E d z=0$ when extended between the two grounded electrodes $z_{1}$ and $z_{2}$ :

$$
\begin{aligned}
\int_{z_{1}}^{z_{2}} D d z & =d_{31} Y_{p}^{E} \int_{z_{1}}^{z_{2}} S d z \\
& =-\frac{\partial^{2} z_{n}(x)}{\partial x^{2}} d_{31} Y_{p}^{E} \int_{z_{1}}^{z_{2}}\left(z-z_{n}\right) d z
\end{aligned}
$$

Since divergence of the dielectric displacement must be null and only the $z$-axis component $D$ is here considered, $D=$ constant. Both integrals in (12) can now be evaluated, leading to:

$$
D=-d_{31} Y_{p}^{E}\left(z_{p}-z_{n}\right) \frac{\partial^{2} z_{n}(x)}{\partial x^{2}}
$$

At each point $x$, the dielectric displacement $D$ is proportional to the curvature $\frac{\partial^{2} z_{n}(x)}{\partial x^{2}}$ which is the only term in (13) which varies along $x$. As in [5], in order to derive the dielectric charge built in the piezoelectric material, the flux of $D$ across the area of one of the electrodes (i.e. $\left.\Sigma=\left[\begin{array}{ll}0 & W_{s}\end{array}\right] \times\left[\begin{array}{ll}0 & L_{a c t}\end{array}\right]\right)$ can be evaluated as:

$$
\begin{aligned}
Q & =\iint_{\Sigma} D d x d y \\
& =-\left.W_{s} d_{31} Y_{p}^{E}\left(z_{p}-z_{n}\right) \frac{\partial z_{n}(x)}{\partial x}\right|_{0} ^{L_{a c t}} \\
& =-W_{s} d_{31} Y_{p}^{E}\left(z_{p}-z_{n}\right) \alpha_{a c t}
\end{aligned}
$$

since the sensor is subjected to the same clamping conditions of the actuator, i.e. cantilevered, and therefore $\frac{\partial z_{n}(0)}{\partial x}=0$ and $\frac{\partial z_{n}\left(L_{a c t}\right)}{\partial x}=\alpha_{a c t}$.

\section{$5 \quad$ Model Limits}

Equation (14) relates the polarization charge built in the sensor directly to the slope $\alpha_{t i p}$ at the end of unimorph actuator and before the extension. A simple way to measure the polarization charge is by reading the voltage that develops across the sensor electrodes when these are left disconnected. In reality piezoelectric materials are affected by several kind of losses, among these the dielectric ones. Considering the piezoelectric layer as a capacitor, if a static charge is initially placed across its electrodes, the capacitor will eventually discharge because of ohmic losses of the dielectric material constituting the capacitor itself. A piezoelectric layer can then be thought of as a pure capacitor $C_{0}$ with a resistance $R_{0}$ in parallel which takes into account the dielectric losses. Elementary circuit theory shows that such a configuration adds a zero in the origin and a pole, i.e. $s /\left(R_{0} C_{0} s+1\right)$, to the final frequency response of the sensor. $R_{0} C_{0}$ represents the discharging time constant of the lossy piezoelectric capacitor.

The pole at $\left(R_{0} C_{0}\right)^{-1}$ is the lower limit for the frequency range at which the sensor provides a flat response. It is not possible to eliminate the zero in the origin, i.e. the sensor cannot be used at DC or in quasi-static conditions, but it is possible to keep the pole at low frequencies by using a charge amplifier, as shown in Fig. 3. This well known circuit pumps an external charge into the sensing electrode in order to neutralize the polarization charge and to keep the voltage across the piezoelectric layer at zero (virtual ground). In this way, since the voltage is virtually zero, there is no effect due to the dielectric losses. In fact, a charge amplifier as the one in Fig. 3 beside the capacitor $C$ has to include a resistor $R$ (not shown in the picture) in parallel. Thus instead of being limited by the $\left(R_{0} C_{0}\right)^{-1}$ the band is limited by $(R C)^{-1}$ which is controllable and can be set to be small enough not to interfere with the frequency range of interest.

\subsection{Band upper limit}

The flat-response band upper limit of the actuator/sensor system is solely given by the first mode of the unimorph actuator. Such a frequency depends upon the geometrical dimensions of the actuator itself which should be designed so that the first resonant mode occurs much after the working bandwidth $(100-200 \mathrm{~Hz}$ for the MFI). Having a flat response simply means that, at any frequency within the flat range, a static model for the actuator is sufficient, i.e. matrices $\mathbf{S}_{\text {act }}$ and $\mathbf{G}_{\text {act }}$ are frequency independent. After the first resonance, a non-flat response of the unimorph actuator could be theoretically considered and $\delta_{\text {tip }}$ and $F_{\text {tip }}$ could still be derived by (7) but $\mathbf{G}_{\text {act }}$ would now be frequency dependent.

\section{Calibration}

Calibration is a necessary step in order to be able to practically use the actuator/sensor device. Both ac- 


\begin{tabular}{|c|c|c|}
\hline$F_{t i p}$ & $V_{p z t}$ & $\delta_{t i p}$ \\
\hline 0 & $150 V$ & $450 \mu m$ \\
$31.3 m N$ & 0 & $200 \mu m$ \\
\hline
\end{tabular}

Table 1: DC measurement for characterizing the stiffness and the free displacement of the actuator.

tuator and sensor calibrations need to be performed but while the former can be done at DC, i.e. with a static procedure, the latter has to be done at $\mathrm{AC}$, i.e. dynamically, since the sensor provides no response at DC.

As also described in [4], by means of an optical microscope, a digital video camera and a TV screen, it is possible to measure linear deflections ranging in the order of $10-10^{3} \mu \mathrm{m}$. For the actuator, described by (6), only $E I$ and $M_{V}\left(M_{p z t}=M_{V} V_{p z t}\right)$ need to be determined since $L_{a c t}$ and $L_{\text {ext }}$ are known. A bending actuator can be characterized at DC by its stiffness and its free displacement. For the former, zero voltage is applied at the electrodes while for the latter no force is applied at the tip. Table 1 reports typical values where $F_{t i p}=31.3 \mathrm{mN}$ is applied by hanging a $3 g$ mass at the tip of the actuator. Measurements in Table 1 are sufficient to calibrate $E I$ and $M_{V}$ from (6) and (8).

Calibration for the sensor has to be performed at AC, i.e. dynamically, within the flat response band. With reference to Fig. 3, given an (AC) input voltage of amplitude $\tilde{V}_{p z t}$, a signal of amplitude $\tilde{V}_{\text {out }}$ can be detected from the charge amplifier and, via optical microscope, the corresponding displacement amplitudes $\tilde{\delta}_{t i p}$ and $\tilde{\delta}_{\text {act }}$ can be measured. The last two measurements are necessary to infer, via (8), $\tilde{\alpha}_{a c t}=\frac{\tilde{\delta}_{t i p}-\tilde{\delta}_{a c t}}{L_{e x t}}$. Frequency response $H(j \omega)=\frac{V_{o u t}(j \omega)}{V_{p z t}(j \omega)}$ of the unloaded actuator can be measured by means of a Dynamic Signal Analyzer (HP3562A). For a given general input $V_{p z t}(j \omega)$, in the frequency domain, $\alpha_{a c t}(j \omega)$ can be evaluated as:

$$
\begin{aligned}
\alpha_{a c t}(j \omega) & =-\frac{\tilde{\alpha}_{a c t}}{\tilde{V}_{\text {out }}} V_{\text {out }}(j \omega) \\
& =-\frac{\tilde{\tilde{\alpha}}_{a c t}}{\tilde{V}_{\text {out }}} H(j \omega) V_{p z t}(j \omega)
\end{aligned}
$$

Equation (15) is justified by the fact that $\alpha_{a c t}$ is related to the polarization charge $Q$ (and therefore to the voltage $V_{\text {out }}$ ) by a linear relationship as in (14). Such a multiplying factor is derived by the calibration constants $\tilde{\alpha}_{\text {act }}$ and $\tilde{V}_{\text {out }}$. The minus sign takes into account the inverting functionality of the charge amplifier in Fig. 3 .
Once $V_{p z t}$ is known and $\alpha_{a c t}$ is derived by (15), $\delta_{a c t}$ and $F_{\text {tip }}$ can be determined from (7) while $\delta_{\text {tip }}$ is derived from (8).

As previously mentioned, the ground shield in Fig. 1 is designed to limit the direct coupling between actuator and sensor. Although greatly attenuated (actuators without such ground shield are affected by a very strong feed-through), it is not completely zeroed, resulting in a small offset signal always in phase ${ }^{1}$ with $V_{p z t}$. Although not perceptible for quantities such as $\alpha_{a c t}, \delta_{a c t}$ and $\delta_{t i p}$, it is evident for $F_{t i p}$ since, when no loading effect is applied at the tip, a non zero force $F_{\text {tip }}=F_{0}$ is measured from (7).

In order to counterbalance such an offset, (7) can be rewritten as:

$$
\left[\delta_{a c t} F_{t i p}\right]^{T}=\mathbf{G}_{\text {act }}\left[\alpha_{a c t}\left(1+\Delta_{o f f}\right) V_{p z t}\right]^{T}
$$

where $\mathbf{G}_{\text {act }}$ is now calibrated (since $E I$ and $M_{V}$ are calibrated) and the constant value $\Delta_{o f f}$ is used to introduce a counterbalancing offset always in phase with $V_{p z t}$. $\Delta_{o f f}$ is chosen in order to zero (or better minimize) $F_{\text {tip }}$ at low frequencies (but still within the flat response frequency range), i.e. canceling out the force offset $F_{0}$.

\section{Testing and Application}

An actuator with an embedded sensor was fabricated as previously described. Its purpose is to be used for driving and sensing, via a 4-bar mechanism (basically a lever), a wing attached at the end of the 4-bar itself as in Fig. 7.

Since nonlinear behaviors are expected when dealing with large angular displacements of the wing (due to the 4-bar mechanism), the fabricated actuator/sensor device was first tested by means of simple point wise mass $m_{t i p}=190 \mathrm{mg}$ attached at the tip of the extension. A point wise mass was chosen in order to match the inertia of the wing as seen before the 4-bar structure, i.e. mimicking the real structure without introducing nonlinearities. The frequency response of such a system was obtained by sweeping, over the frequency range of interest for the MFI, a driving signal $V_{p z t}$ of constant amplitude $10 \mathrm{~V}$ peak-to-peak and by

\footnotetext{
${ }^{1}$ Since the circuit in Fig. 3 is meant to measure a charge, any parasitic capacitance $C_{\text {par }}$ between the actuating electrode and the sensing one will generate a charge $Q_{\text {par }}=C_{\text {par }} \Delta V$, where $\Delta V$ is the voltage between the two electrodes. Because of the operational amplifier, the sensing electrode is kept at virtual ground, i.e. $\Delta V=V_{p z t}$. $Q_{p a r}$ is then proportional to $V_{p z t}$ and therefore always in phase with it.
} 

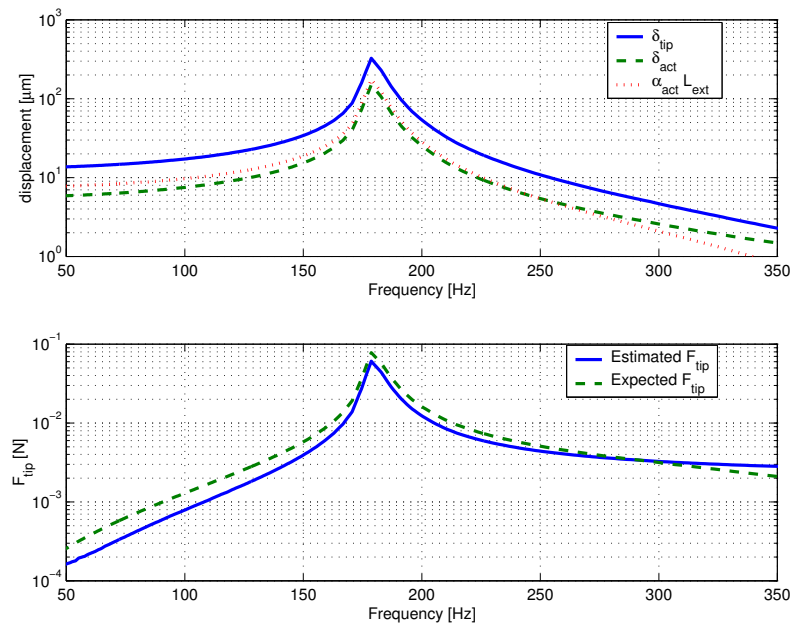

Figure 6: Amplitude versus frequency plots of (above) $\alpha_{a c t}, \delta_{a c t}, \delta_{t i p}$ and (below) $F_{t i p}$ estimated and expected.

reading the output signal $V_{\text {out }}$ out of a charge amplifier as the one in Fig. 3 (a $10 n F$ capacitance was used with a $5 M \Omega$ in parallel, leading to an expected lower band limit of about $3 \mathrm{~Hz}$ ). By using (15) and (16), i.e. after calibration, $\delta_{a c t}$ and $F_{t i p}$ can be estimated and therefore $\delta_{t i p}$ is also derived from (8). $\alpha_{a c t} L_{e x t}$, $\delta_{a c t}$ and their sum $\delta_{\text {tip }}$ are shown in the upper plot in Fig. 6. In the lower plot the estimated $F_{t i p}$ and its expected value are compared, showing fairly good matching. The expected value corresponds to the inertial force due to $m_{t i p}$, i.e. the mass itself times the second time derivative of $\delta_{t i p}$ (acceleration of $m_{t i p}$ ), which in the frequency domain can be expressed as $(j \omega)^{2} \delta_{t i p} m_{t i p}$. For $\delta_{t i p}$ the estimated value was used since it agreed well with what was measured via the optical microscope at various frequencies.

The test just described shows that the sensor behaves accordingly to the model when the actuator is subjected to operating conditions of interest for the MFI. It can now be tested with the MFI mechanical structure.

\subsection{Application to the MFI}

A simple diagram of the thorax of the MFI is shown in Fig. 7. A 4-bar mechanism is used as a mechanical transformer to convert the force and linear displacement of the actuator into the torque and the angular displacement needed to drive the wing. Large forces are transformed into small wing torques which are difficult to directly measure without wires

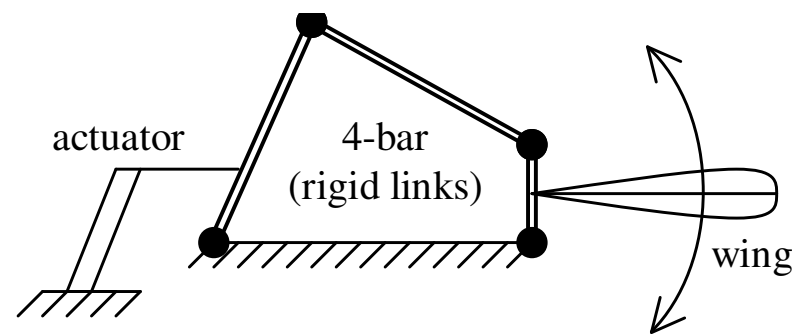

Figure 7: Simplified diagram of the MFI thorax comprising actuator, 4-bar (lever) mechanism and flapping wing.

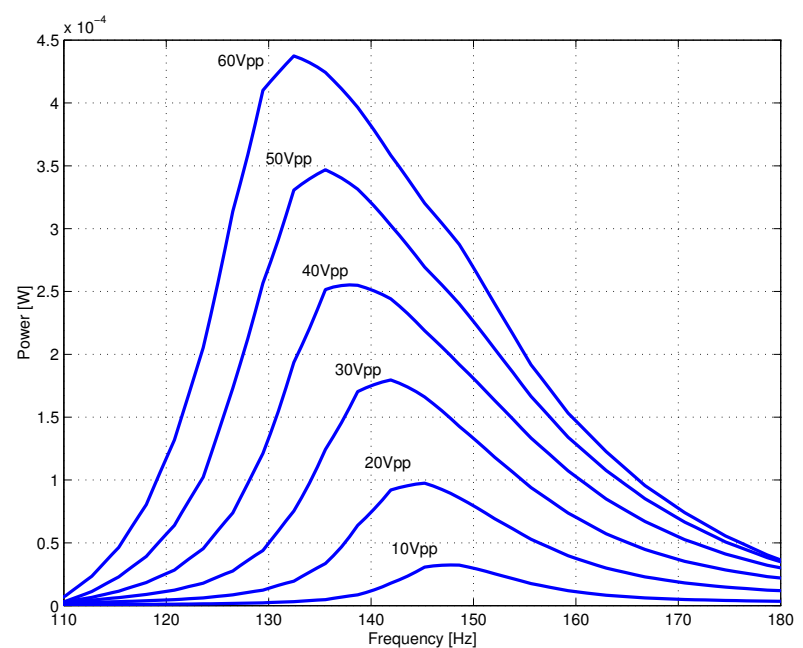

Figure 8: Power dissipated by the wing for incremental driving voltage.

on wings. Sensors are more conveniently placed on the actuating section, and the wing angle is inferred by knowing the 4-bar amplification ratio (although nonlinear). Fig. 8 shows, for increasing input voltage, the mechanical output power dissipated by the wing $\frac{1}{2} R e\left[v_{t i p}(j \omega) F_{t i p}^{*}(j \omega)\right]$ provided by the actuator, where $v_{t i p}(j \omega)=j \omega \delta_{\text {tip }}(j \omega)$ is the velocity of the actuator tip (in the frequency domain) and the supercript $^{*}$ denotes the complex conjugate. Both $v_{t i p}(j \omega)$ and $F_{t i p}(j \omega)$ have been estimated from the input voltage $V_{p z t}$ and the sensor output.

Fig. 8 also shows a typical nonlinear behavior, i.e. the resonant frequency shifts towards lower frequencies when the driving voltage amplitude is increased. The actuator, which is linear at the operating conditions of the experiment, is transformed into a nonlinear one by the saturation of the 4-bar transmission 
ratio. In particular, a softening of the transformed actuator occurs, i.e. for larger displacement the actuator becomes more compliant. It can be shown, [3], that a damped mass-spring system with a softening spring leads to frequency shift as the ones shown in Fig. 8.

\section{Summary and Future Work}

By patterning the electrodes of a piezoelectric layer by means of a low power laser beam, sensing capabilities were embedded into a standard piezoelectric unimorph actuator. In order to reduce the electromechanical coupling between actuating and sensing section an additional electrode was patterned and grounded providing a shielding effect that significantly reduced the coupling. A model was derived for the sensor which, together with the static model of the actuator, provided a simple means of estimating external force and displacement at the tip of the actuator itself. The calibration steps were outlined in order to practically use a fabricated device. A point wise mass was attached at the tip of the actuator and its weight was chosen in order to reproduce the operating conditions arising when an wing is driven, through a coupling mechanism, by the actuator. The force measured by the sensor matched the expected ones which were computed by perfectly knowing the nature of the load, i.e. the point wise mass. The actuator/sensor device was then used to drive a MFI thorax and the sensor signal was processed to derive the mechanical power fed into the wing, an important information for wing flapping mechanisms. As future work, the actuator/sensor device will be used to induce the wing to track the desired kinematics.

\section{Acknowledgments}

The authors would like to thank Srinath Avadhanula, Gabe Moy, Metin Sitti, Joe Yan and Robert J. Wood for useful discussions and assistance.

\section{References}

[1] R.S. Fearing, K.H. Chiang, M. Dickinson, D.L. Pick, M. Sitti, and J. Yan, "Wing Transmission for a Micromechanical Flying Insect," Proc. of the IEEE Robotics and Automation Conf., pp. 15091515, San Francisco, CA, USA, April 2000.

[2] C. K. Lee, "Piezoelectric Laminates: Theory and Experiments for Distributed Sensors and Actua- tors," Intelligent Structural Systems, Kluwer Academic Publishers, pp. 75-167, December 1992.

[3] S. Sastry, Nonlinear Systems: Analysis, Stability, and Control, Springer-Verlag, New York, 1999.

[4] M. Sitti, D. Campolo, J. Yan, R.S. Fearing, T. Su, D. Taylor, and T. Sands, "Development of PZT and PZN-PT Based Unimorph Actuators for Micromechanical Flapping Mechanisms, " IEEE Int. Conf. Robotics and Automation, pp. 3839-3846, Seoul Korea, May 21-26, 2001.

[5] J. G. Smits and A. Ballato, "Dynamics Admittance Matrix of Piezoelectric Cantilever Bimorphs," Journal of Microelectromechanical Systems, pp. 105-112, vol. 3, 1994.

[6] Kenji Uchino, Piezoelectric Actuators and Ultrasonic Motors, Kluwer Academic Publishers, Boston, 1997.

[7] M. Weinberg, "Working equations for piezoelectric actuators and sensors," Journal of Microelectromechanical Systems, vol. 8, pp. 529-533, Dec. 1999. 